Dicenda. Cuadernos de Filología Hispánica

ISSN: 0212-2952

http://dx.doi.org/10.5209/DICE.57710

\title{
Reescrituras celestinescas musicales: apuntes para una historia del libreto celestinesco ${ }^{1}$
}

\section{Laura Mier Pérez ${ }^{2}$}

Resumen. En este trabajo ofrecemos una visión de las adaptaciones musicales que se han hecho de la Tragicomedia de Calisto y Melibea bajo el formato de la ópera o de teatro musical. Retomando los trabajos de Joseph Snow proponemos un análisis de la adaptación de Felipe Pedrell de principios del siglo XX y llevamos a cabo un estudio de la última versión conocida, la que se está llevando a cabo en el Metropolitan Museum of Art de Nueva York de la mano de Patrick Eakin Young. Aunque tenemos en cuenta todas las adaptaciones conocidas, nuestro trabajo se centra principalmente en las dos indicadas, con especial atención a la última, de la que carecemos por completo de estudios previos.

Palabras clave: Celestina; ópera; teatro; adaptaciones.

\section{[es] Musical Rewrites Based on Celestina: Notes on the History of Celestinesque Libretto}

\begin{abstract}
In this paper we offer a vision of the musical adaptations made from the Tragicomedia de Calisto $y$ Melibea in the format of opera or musical theatre. We take into consideration the previous works by Joseph Snow to propose an analysis of Felipe Pedrell's adaptation from the beginning of the $20^{\text {th }}$ century as well as the last known version, the one that is taking place in the Metropolitan Museum of Art of New York directed by Patrick Eakin Young. Even though we take into account all the known adaptations, our paper focuses in the two referred above, with a special attention to the latter given the lack of study about this text.
\end{abstract}

Keywords. Celestina; opera; theatre; adaptations.

Cómo citar: Mier Pérez, L. (2017). Reescrituras celestinescas musicales: apuntes para una historia del libreto celestinesco, Dicenda. Cuadernos de Filología Hispánica, 35, 247-258.

La importancia de la Tragicomedia de Calisto y Melibea como clásico literario es hoy indiscutible y ocupa uno de los primeros lugares del canon que conforma nuestro patrimonio literario junto a obras como el Quijote Una de las afortunadas consecuencias inmediatas de esta consideración es que el texto de Rojas se ha convertido en una inspiración constante para los artistas, no sólo en el ámbito de la literatura. En este trabajo queremos trazar una pequeña historia de La Celestina como fuente

\footnotetext{
1 Este trabajo ha sido posible gracias a la financiación del proyecto TESAL 16. Documentación, edición, estudio y propuestas de representación del teatro del siglo XVI en Salamanca dirigido por Javier San José Lera (SA 155A-11-1).

2 Universidad de Cantabria \& IEMYR laura.mier@unican.es
} 
de inspiración, desde el círculo de recepción más inmediato; las adaptaciones, traducciones e imitaciones del siglo XVI, para llegar a las versiones musicales que tienen lugar en el siglo XX y XXI; especialmente las incluidas bajo el género de música escénica o teatro musical. Es decir, queremos constatar cómo el éxito inicial de la Tragicomedia sobrevive a lo largo de los años con la vitalidad asombrosa de convertirse en un referente fundamental en la ópera. Con este artículo pretendemos, pues, sentar la primera piedra para poder reconstruir la historia del texto celestinesco como libreto literario y sus sucesivas transformaciones. No pretendemos en ningún caso ofrecer un estudio terminado.

El éxito de la Comedia de Calisto y Melibea de Fernando de Rojas es indiscutible desde la misma fecha de edición, 1499. Se suponen ediciones de la Comedia en Burgos, 1499; Salamanca, 1500 y Toledo, 1500. El gusto por la obra es tal en los tres primeros años de vida que el propio Rojas se ve en la tesitura de ampliar su modelo. Así, la Tragicomedia fue editada en Roma, 1506 y en Zaragoza, 1507; Valencia, 1514; Sevilla, 1518; Toledo, 1526; Salamanca, 1570, etc. A pesar de no tener un número exacto de ediciones durante el siglo XVI las siguientes palabras de Emilio Blanco (2001: 19) pueden ayudarnos a tener una idea de las dimensiones del fenómeno:

Habría que comenzar señalando el inmenso éxito editorial de la Celestina durante el siglo XVI, que multiplica las ediciones y las traducciones, y que por ende acumula ejemplares en los almacenes de los libreros e impresores españoles. Baste decir, por ejemplo, que entre 1501 y 1560 el libro se edita con frecuencia bianual, con un monto de 32 ediciones. Cifra que J. H. Herriot hacía ascender hasta 187 ediciones en todo el siglo XVI. Sin entrar ahora a discutir la veracidad del último dato, lo que es innegable es la pertenencia al grupo de best-sellers del siglo XVI, en el que ocupa además el primer lugar.

Blanco y Montoya (1979) rastrean la presencia de ejemplares de Celestina en los inventarios de libreros. A pesar de ser un campo del cual no conservamos la suficiente información como para poder realizar una historia editorial precisa, sí se puede percibir, con la muestra que tenemos que "el texto de Rojas formaba parte del fondo editorial de cualquier librero" (Blanco, 2001: 19)3.

El éxito editorial no se limita al territorio hispanohablante; Brault documenta tanto la primera traducción en verso al inglés en 1530 (muy anterior a la famosa de James Mabbe de 1631) como la presencia celestinesca en Inglaterra durante el siglo XVI (Brault, 1960). Snow (1997) traza una historia de las versiones en italiano que comienzan en 1506; en alemán en 1520, y en francés y en hebreo en 1527. En Portugal McPheeters (1977) rastrea la presencia de La Celestina en la obra de Gil Vicente, que fecha en 1511, con la escritura del Auto das fadas. De hecho, veremos que la gran mayoría de las óperas tienen lugar también fuera de la Península Ibérica.

\footnotetext{
Algunos de los datos que proporciona son los siguientes: a la muerte de Jacobo Cromberger en 1528 se inventariaron en sus estancias 1401 Calistos. En 1540 entre los libros de Juan Cromberger, se encuentran 262 Calistos y 63 Celestinas En Salamanca, en 1557 entre los libros de Juan de Junta se documentan 30 Celestinas. En Granada, en 1571 a la muerte de Martín Salvatierra, hay 8 ejemplares; 39 en la de Francisco García en 1583. En Medina, Benito Boyer, en 1592 hay 27 y, en 1606, en la de Cristóbal López, 4.
} 
En 1989 el "descubrimiento" por Charles Faulhaber del Manuscrito de Palacio deja ver una difusión no editorial sospechada hasta el momento, pero no documentada. Este testimonio ha tenido un gran impacto en la crítica y ecdótica celestinesca ya que ha supuesto un cambio considerable en la historia de la transmisión del texto ${ }^{4}$. Uno de los principales puntos de conflicto ha sido establecer la naturaleza de esta copia manuscrita y su utilidad; asunto que parece no estar cerrado aún. Sin embargo, independientemente de cuál sea la función de este testimonio podemos considerar que (Conde, 1997: 185):

El alto número de errores de copia, puramente mecánicos, la deficiencias en la transcripción de nombres propios y muchos otros defectos que se alternan en nuestro manuscrito, con lecturas llamativas por su calidad textual hacen preciso postular una gran vitalidad en esta rama de la tradición textual de la Celestina, rama que podemos considerar sin grave riesgo de error representación de una fecunda -aunque breve en el tiempo, desplazada pronto por la tradición impresa- transmisión de la obra, de la cual sólo nos queda este representante.

Es decir, la amplia difusión de La Celestina es, al día de hoy, indiscutible, y así lo reflejan los estudios que, como hemos visto, contemplan los diferentes aspectos que nos permiten reconstruir la historia del texto: libreros, transmisión impresa y transmisión manuscrita. No es de extrañar, pues, que esta profusa difusión pronto se convierta en fuente de inspiración:

A work of this magnitude could hardly have existed only to its pure form with this intensity for a century and a half. The refreshing newness of this work impacted significantly on the imagination of not only readers bur also of authors, anonymous and otherwise, almost from the outset (Snow, 2000:4).

Por un lado, pronto surgen "imitaciones" teatrales del original que acaban convirtiéndose en un género propio; aquél que Menéndez Pelayo (1943) dio en llamar celestinesca ${ }^{5}$. Sin embargo, lo que nos interesa especialmente son las obras no teatrales que también comienzan a surgir en fechas muy próximas a las primeras ediciones de La Celestina. Por ejemplo, Menéndez Pidal data un Romance de Calisto y Melibea hacia 1510 en su trabajo de 1952, Pedro Manuel Ximénez de Urrea publica su versificación en 1513 y en 1540 y Juan de Sedeño saca en metros castellanos la prosa de la Tragicomedia (Snow, 1997). En poco tiempo nuestro clásico ha pasado de ser imitado a ser fuente de inspiración y su naturaleza genérica ha empezado a evolucionar.

Las producciones que toman nuestro texto como referencia continúan a lo largo del siglo XVI. Sin embargo, el éxito inicial comienza a decaer en el ámbito de la producción artística, si bien es cierto que, como documenta Snow a través de referencias, el interés por nuestra obra se mantiene durante siglos, a pesar de dejar de ser un texto de consumo inmediato (Snow, 1997b). Este interés latente vuelve a ser evidente con la publicación en 1822 de la primera edición moderna en Madrid, de la

Véanse, entre otros, Faulhaber, (1990 \& 1991); M. Garcia, (1994); F. Lobera Serrano, (1993); P. Botta, (1993), (1995 \& 1997); J. Conde, (1997)

5 Tanto la nómina de obras como las características de las mismas han sido reseñadas en numerosas ocasiones, razón por la cual no consideramos necesario un tratamiento más profundo en este artículo. 
mano de León Amarita. Durante el siglo XIX se produce una vuelta a La Celestina que derivará en productos artísticos exquisitos, así como en el inicio de una tradición crítica que nos lleva al día de hoy.

El impacto literario de la Tragicomedia es incuestionable; tanto de forma inmediata como a lo largo del tiempo, como acabamos de ver. Sin embargo, uno de los aspectos que más llama nuestra atención es precisamente el uso que de Celestina hacen otras artes. Todos estamos familiarizados con representaciones pictóricas e incluso escultóricas, por ejemplo. Pero una de las manifestaciones más curiosas es aquella que se da en el ámbito musical. El marco de la ópera, que facilita la fidelidad al texto y respeta la plasticidad del discurso dramático, proporciona, al mismo tiempo, otra expresividad añadida que es el lenguaje musical. El interés decimonónico por nuestro clásico, vivido tanto en círculos intelectuales como artísticos, tiene, en cierta medida, la culminación de este proceso en La Celestina. Tragicomedia lírica de Calisto y Melibea de Felipe Pedrell, compuesta en 1902, que es, a su vez, la primera ópera de tema celestinesco de la que tenemos noticia ${ }^{6}$.

Del proceso creativo que llevó a Pedrell a emprender esta obra se ha ocupado con detalle Snow (1979), basándose en gran medida en las declaraciones que el propio autor recoge en su libro Orientaciones (1909). Cabe destacar que tanto el libreto, en cuatro actos, como la música son del mismo autor y que el proceso de composición fue parejo al de redacción ${ }^{7}$. A pesar de no haberse estrenado nunca en su totalidad (Casalls estrenó algunos fragmentos en octubre de 1921 en el Palau de la Música Catalana como homenaje al compositor, que cumplía ochenta años) es una obra de gran trascendencia para la historia de la música, ya que Pedrell, maestro de maestros, introduce elementos populares dentro de la música clásica, renovando, de esta forma, la manera de concebir manifestaciones artísticas como la ópera y poniendo las bases del gran fenómeno de recuperación y creación musical que llevaron a cabo maestros como Albéniz o Falla, -este último discípulo directo de Pedrell-. Del libreto, del que pronto publicaremos un estudio más detallado, cabe destacar un respeto reverencial hacia el texto de Rojas y una sensibilidad exquisita a la hora de comprenderlo. Afortunadamente éste sí fue editado por el propio autor y podemos consultarlo fácilmente en 1903. El hecho de que Pedrell mismo decidiera publicar el libreto como texto independiente nos permite hacernos una idea del grado de implicación que tenía en este proyecto y de la importancia que concedía a las palabras de Rojas.

En el ámbito español el otro gran intento de convertir la obra de Calisto y Melibea en una ópera lo lleva a cabo Joaquín Nin-Culmell en 1995, fecha en la que se edita la partitura en París. Esta obra fue un proyecto que acompañó al compositor durante toda su vida y en el que también se encargó tanto del texto como de la música. La

Aparte de los estudios específicos de cada género para el que escribió Pedrell, su obra se ha visto desde diversas perspectivas, pero desde luego, la redacción del manifiesto Por nuestra música en 1891 ha marcado en buena medida nuestra percepción actual del autor. A pesar de no ser el objetivo de este trabajo, no podemos evitar reseñar algunos de los estudios que nos acercan más a la idea que Pedrell tenía de la música. Para comprender su visión de las zarzuelas, operetas y, sobre todo, óperas (entre las que podemos incluir los arreglos de ópera y los poemas líricos), es de gran utilidad lo propone Bonastre i Beltrán, 2001. Respecto a la producción operística resultan también muy útiles los siguientes trabajos de F. Cortés, 1991-1992, 1996 \& 2004-2005. Para la importancia de Pedrell dentro de la historia de la música española podemos consultar: Gregori, 1991-1992; Martín Moreno, 1991-1992 y Bonastre, 2004-2005, entre otros.

7 Agradezco a Victòria dels Àngels Pérez de L'Auditori de Barcelona que me haya hecho llegar una copia de la grabación que se hizo en 1999 con parte de la obra de Pedrell. 
primera versión la realizó entre los años 1956 y 1959 para revisarla posteriormente en 1985 y 1991 ante la posible circunstancia de un estreno. Cuenta Gereben que el compositor quemó las dos primeras versiones para que nadie pudiera decidir por él cuál era la mejor cuando muriera.

Del texto podemos decir que son tres actos y seis escenas acompañadas de un prólogo solamente musical, en el que se narra, sin palabras, el primer diálogo entre Calisto y Melibea, reflejado en el libreto mediante la siguiente acotación: "Prólogo: el encuentro". Cualquier adaptación requiere una profunda interpretación y en el libreto de Nin-Culmell podemos comprobar cómo desde el mismo dramatis personae es así ${ }^{9}$. En el esfuerzo de reducir el texto al máximo (el libreto apenas ocupa dieciséis páginas) Nin-Culmell recurre a la condensación de motivos en un solo parlamento y a la eliminación evidente de muchos. Es efectivamente el principal problema que presenta la adaptación operística de Celestina. El texto en sí es excesivo para el gusto contemporáneo y más aún cuando el discurso textual necesita más tiempo para desarrollarse dentro del musical. Otra de las cuestiones derivadas del género operístico es la obligatoriedad de repetir algunos sintagmas que en apariencia son superficiales si contemplamos sólo el texto para favorecer el discurso musical ${ }^{10}$. En este proceso de eliminación hay momentos de difícil comprensión, como apuntaba Gimber que parecen forzar al espectador a conocer la trama de antemano ${ }^{11}$. Por ejemplo, el primer acto se cierra en casa de Celestina; ahí tienen lugar dos escenas. En la primera, Celestina se acerca a Areúsa y la contempla desnuda, escena que no tiene sentido sin la presencia escondida de Pármeno, que no está en la ópera. Inmediatamente después, sin transición ninguna, Celestina ofrece el conjuro a Plutón. Otro ejemplo claro es el proceso por el cual Celestina convence a Melibea, que fusiona los autos cuarto y décimo de la Tragicomedia dando como resultado una rápida transición en el carácter de Melibea y una pérdida clara del poder dialéctico de la Puta Vieja; sin duda, una de las delicias literarias del original.

Otro de los grandes problemas de adaptación textual que presenta la ópera es la de la participación del coro, fundamental en cualquier espectáculo de este tipo, como portador de una voz colectiva, y no previsto en absoluto en el original de Rojas. Nin-Culmel lo soluciona añadiendo dos intervenciones. Una tiene lugar cuando Celestina consigue concertar la primera (y única) cita con Calisto, al final de la primera escena del segundo acto. Para ello utiliza la primera estrofa de la conocida composición que Juan del Encina compuso a la muerte del príncipe Juan, "Triste España sin ventura", cambiando el asunto de "España" a "amante". La segunda vez tiene lugar en la escena final: cuando muere Calisto el coro comenta la trágica escena ${ }^{12}$. En otros fragmentos hace cantar a modo de coro a todos

Agradezco la generosidad de Javier San José, de la Universidad de Salamanca; Almudena Pedrero, del Teatro de la Zarzuela y de Ignacio García, director de escena del montaje del estreno, por haberme hecho llegar el libreto, que no ha sido editado, y los materiales necesarios para conocer esta obra.

9 Los personajes aparecen definidos por un epíteto caracterizador: Celestina, alcahueta; Calisto, mancebo enamorado; Melibea, objeto de su amor; Sempronio, sirviente de Calisto; Pármeno, sirviente de Calisto; Areusa, ramera; Elicia, ramera; Lucrecia, sirvienta de Melibea; Socias, sirviente de Calisto; Pueblo, coro mixto.

10 Por ejemplo, dice Calisto: "Recibid la dádiva de aquél, de aquél / que con ella, con ella, con ella / la vida ofrece, la vida ofrece, la vida ofrece / la vida, la vida, la vida".

A. Gimber, 2009.

12 Son tres las intervenciones en total y todas son añadidos que no están en el texto original: "A tal pérdida tan triste [sic]", "Buscarle consolación [sic]", "Claro está que es traición" 
los personajes que están en el escenario a la vez, que se oponen, por lo general, a Celestina ${ }^{13}$.

Entre las modificaciones más significativas cabe señalar el hecho de que Melibea cante una canción popular catalana, La Pastoreta y una intención de final abierto, en el que Melibea no llega a suicidarse en escena, aunque sí a cantar su intervención conclusiva en la que declara sus intenciones ${ }^{14}$.

En cuanto a la música, cabe destacar que tanto Miguel Ortega (responsable musical del estreno) como Tomás Marco destacan que se trata de una versión historicista, que respeta la armonía y la melodía y que no recoge las últimas tendencias de composición musical, es decir, se trata de un planteamiento musical ajeno a las vanguardias y que intenta, de alguna medida, rendir homenaje al momento musical de la concepción de Rojas, como hemos podido ver, por ejemplo, en el caso concreto del coro.

La obra iba a estrenarse, finalmente, en el Liceu de Barcelona, pero el gran incendio de 1994 obligó a suspender la programación. Tras varios intentos frustrados la obra fue estrenada en el Teatro de la Zarzuela de Madrid el 19 septiembre de 2008, cuatro años después de que muriera el compositor y para conmemorar el centenario de su nacimiento ${ }^{15}$.

Fuera de nuestras fronteras en 1961 Flavio Testi edita la partitura para piano y voz de su ópera La Celestina. Opera in tre atti dalla tragicommedia omonima di Fernando de Rojas que fue estrenada en el Teatro della Pergola en Florencia el 28 de mayo de 1963. En 1970, Karl-Heinz Füssl compone una ópera en dos actos con libreto de Herbert Lederer que se estrenó en el teatro de Karlsruhe en 1976.

13 Por ejemplo, en la tercera escena del segundo acto: "CELESTINA: Ponéos en orden, cada uno cabe de lo suyo / TODOS: Cada uno lo suyo, cada uno lo suyo".

14 La última intervención de Melibea dice así:

¡Oh, la más triste de las tristes!

¡Tan tarde alcanzo el placer,

Tran presto venido el dolor!

¡Ay, dolor! ¡Rezando

llevan mi bien, mi todo!

¡Muerta, muerta mi alegría!

Mi fin es llegado;

llegado es mi descanso;

llegado es mi alivio;

llegada es mi acompañada hora.

Me acortaron la esperanza,

me acortaron mi gloria,

me acortaron mi vida.

¡Oh, Calisto, mi vida!

¡Oh, Calisto, mi muerte!

Espera, espera, espérame, ya voy

15 En el estreno los artistas fueron los siguientes: Celestina: Alicia Berri; Calisto: Alain Damas; Melibea: Gloria Londoño; Sempronio: José García-Quijada; Pármeno: Andrés del Pino; Areúsa: Carolina Barca; Elicia: Soledad Cardoso; Lucrecia: Belén Elvira; Miguel Angel Bustamante: Socias; Coro del Teatro de la Zarzuela de Madrid; Maestro del Coro: Antonio Fauro; Orquesta de la Comunidad de Madrid; Director Musical: Miguel Ortega; Directora Artística: Ana María Iriarte.

Podemos consultar los bocetos adaptados por Domenico Franchi sobre los diseños de vestuario de Lluís Juste de Nin, que fueron utilizados en el estreno en: http://www.mcu.es/novedades/2008/novedades_LaCelestina. html.

Una reproducción de la escenografía de Domenico Franchi está disponibles en: http://www.behance.net/gallery/ LA-CELESTINA-di-Joaquin-Nin-Culmell/8508431. 
En 1979 Jerome Rosen, fundador del Departamento de Música de la Universidad de California en Davis, compone una ópera en tres actos y dieciséis escenas con el título de Calisto \& Melibea. A Comedy of Love, Seduction and Deathy libreto de Edwin Honig. La obra, con sus 3 actos y dieciséis escenas se distribuye en cuatro escenografías: el jardín de Melibea, las habitaciones de Calisto, la casa de Celestina y el interior de la casa de Melibea. Curiosamente el texto en el cual se basa Honig para elaborar el libreto es la traducción de James Mabbe de 1631. La obra fue estrenada en Davis, California, el 31 de mayo de 1979 y Anderson nos ofrece una cuidadosa reseña de la puesta en escena, de la que destaca que "the musical style, the set design, the costumes and the lighting all are carefully designed and coordinated for an expressionistic emphasis on the symbolic representation of mood and emotion" (Anderson, 1979:30) ${ }^{16}$.

Por último, Maurice Ohana compuso música y libreto (éste último con la ayuda de Odile Marcel), de la obra titulada La Celestina. Tragicomedia lírica basada en Fernando de Rojas en dos actos y once escenas con prólogo y epílogo. Fue estrenada en París, en el Palais Garnier el 13 de junio de 1988. La concepción de Ohana se caracteriza por su marcado carácter político, ya que la sitúa en 1492, e inserta dos cuadros de contenido histórico: la entrada en Granada de los Reyes Católicos y la vuelta de Colón. También reduce los personajes y acorta la trama a dos horas aproximadamente. Como dato curioso cabe señalar que el propio Rojas sube al escenario bajo el nombre de "El Iluminado" para comentar el sentido de la obra y que el texto es plurilingüe.

Gracias a Snow sabemos que hubo más proyectos de este tipo (Snow \& Gimber, 2007). Así, p, , arece que en 1962 Federico Romero adaptó el texto en verso con la esperanza de que Oscar Esplá le pusiera música, aunque todo apunta a que esto no llegó a suceder y pasó a formar parte de las composiciones truncadas del músico. También recoge la posible Celestina que Ángel Arteaga compusiera entre 1966 y 1068, aunque la única comprobación es a través de referencias, sin haber podido verificar la existencia del texto en sí (Snow \& Gimber, 2007:161). Pero sin duda, el proyecto más interesante de todos los frustrados es el que parece que quiso llevar a cabo Richard Strauss entre 1935 y 1936. Según el proceso epistolar que Snow reconstruye, 1, la idea original parte de Stefan Zweig y pronto se convierte en un intercambio de impresiones entre los dos sobre el potencial operístico de la obra (Snow \& Gimber, 2007). Cuentan también con Joseph Gregor, historiador, como asesor. Desgraciadamente, lo único que nos queda de todo este plan de creación es un esquema de diez páginas para los tres actos (cuyo texto edita Joseph Snow en el trabajo anteriormente mencionado), además de las cartas que atestiguan el intercambio de ideas entre Zweig y Strauss.

El último proyecto del que hemos tenido noticia se desarrolló durante la primavera pasada en el Metropolitan Museum of Art de Nueva York y lo lleva a cabo la compañía basada en el Reino Unido llamada Opera Erratica. Se trata de una ópera con elementos pregrabados que tiene una duración de unos 30 minutos y que utiliza música árabe, judía y cristiana de los siglos XIII y XIV. Podemos decir que se trata de una ópera-instalación que se sitúa argumentalmente después de la muerte de los

16 Curiosamente el aspecto que menos le gusta a Anderson es el del registro expresivo en el que se escribe la ópera, ya que le parece que no se respeta convenientemente el aspecto de "tragicomedia" y se queda excesivamente en la parte de "comedia", confiriendo un matiz, en ocasiones, excesivamente superficial. 
protagonistas y tiene lugar en la plaza pública, tanto física como metafóricamente, donde confluyan diferentes voces (estructura inspirada en Rashomon de Akira Kurasawa) que comenten la "tragedia de Calisto y Melibea". El público participa activamente paseándose por esta "plaza" en la que se habla de la fortuna de los amantes (http://opera-erratica.org/projects/celestina, página web consultada el 2 de noviembre de 2014 $)^{17}$. Esta performance integra diferentes recursos artísticos, como las marionetas, las vídeo- instalaciones diseñadas por Manual Cinema y la música de Matt Rogers ${ }^{18}$.

En el caso de esta ópera la distancia entre el texto original de Rojas y el que es puesto en voz de los cantantes es muy grande, ya que se trata de una lectura libre de La Celestina. Patrick Eakin Young, responsable del libreto, mezcla personajes que han sobrevivido a la tragedia, como Areúsa; personajes históricos, como Felipe II; personajes alegóricos: Otoño, Primavera y Templanza; personajes de la mitología, Alfeo y Fauno con un César, un Emperador y tres niños en escena. El resultado de esta combinación es una serie de voces plurales con puntos de vista muy diferentes sobre lo que ha sucedido. Como señalábamos más arriba, la acción comienza cuando ya se ha producido la tragedia y lo que se intenta recoger son las opiniones que ha generado en la sociedad.

Es posible, sin embargo, reconocer en algunos momentos fragmentos tomados del original de 1499. Para empezar, los personajes a los que se refieren aparecen caracterizados según los atributos de Rojas, especialmente los tres protagonistas: Celestina, Melibea y Calisto. Aunque también son perfectamente reconocibles Pármeno y Sempronio en su definición. Los personajes alternan sus intervenciones y en muchas ocasiones son simultáneas, no obstante, podemos rastrear un diálogo entre ellos aunque no sea al modo más tradicional de la ópera, es decir, no tiene lugar de forma sucesiva, sino sincrónica. De hecho, la obra se abre con la expresión simultánea de diferentes sentimientos al respecto ${ }^{19}$. A iniciativa del Emperador comienzan a recordar la historia manteniendo con fidelidad algunos detalles: las cien monedas de pago a Celestina y la forma de convencer a Pármeno para que se uniera al plan de conquistar a Melibea, entre otros. Así como frases

Agradezco al director artístico, Patrick Eakin Young, toda la información que me ha brindado sobre este proyecto, así como la amabilidad y disposición de Miriam Sherwood y Liam Byrne, cuya ayuda ha sido imprescindible para poder escribir estas páginas.

18 Pueden consultarse fragmentos del montaje en https://vimeo.com/119237979, http://abc7ny.com/society/singing-sculptures-at-the-met/569707/.

19 Nos tomamos la libertad de reproducir esta primera escena para que el lector pueda hacerse una idea de cómo está escrito el libreto. Al concluir la exclamación inicial, todos los personajes cantan la palabra "Love" al mismo tiempo.

Autumn:

Spring: Ay! Ay! Life is so full of anguish! Nonsense!

Emperor: Ay! Ay! What devastation! Who would see it coming?

Ceasar: Ay! Ay! So sad, so sad. Who saw it happen?

Philipp II: Ay! Ay! What a disaster... So beset my misery! More dangerous than the Devil.

Temperance: I grind... Water in the mortar. Nobody has mercy on me. Me, the stranger... Me, the stranger.

Alpheus: Ay! Ay! How could this be! Unbelievable...

Arethusa: Oh false world! Who knew it was possible...

Faun: Ay! Ay! Who is to blame? More dangerous than the Devil?

Children: Ay! Ay! It was the Devil! It was love.

Todas las citas, incluidas las de las canciones populares que se detallan a continuación, están tomadas de la partitura que nos ha facilitado el equipo artístico de la compañía. 
prácticamente literales que nos ayudan a imbuirnos en el universo celestinesco: "Who is this woman rustling her skirts?", dice Felipe II, o la referencia a que Calisto muere sin confesión.

No cabe duda de que el personaje más desarrollado y más basado en el original es el que da título a la instalación operística, Celestina. Se mantiene su edad, reiterando lo vieja y arrugada que está y se recuerda la inseguridad que sentía al acercarse a casa de Melibea, incluso sintiéndose acompañada por el conjuro demoníaco, que repite Templanza: "Curse the hour I came here if my spell fails. Can you hear me spirit? A storm soon passes... Troy was mightier and I've tamed more fiery souls". También se la define como maga, con referencia específica al hilado.

Por otro lado, se puede contrastar la lectura individual del clásico que el autor ha realizado, tomando partido en algunos de los tópicos que han enzarzado a los estudiosos del renacimiento. Dice Otoño, por ejemplo: "She could never have done it if Melibea wasn't already in love". La idea queda reforzada, más adelante, no dejando ninguna duda sobre la postura que Young ha tomado respecto al enamoramiento de Melibea y, consecuentemente, la eficacia de la magia cuando escuchamos la voz de la soprano Areúsa: "She'd fallen in love with him. From the moment she saw him..."

La clara voluntad polifónica a la que nos referíamos con anterioridad y que, en cierta medida, deriva de la inspiración original, queda muy clara cuando los tres niños preguntan: "But why did he [Calisto] fall?" a lo que cada personaje responde una cosa: por su deseo temerario, por ser un accidente, como resultado de un castigo del destino, se trata de una coincidencia, o fueron Elicia y Areúsa las que lo mataron intencionadamente.

Por último, en cuanto a las relaciones textuales con la Tragicomedia cabe señalar que hay pasajes en los que se ponen en boca de los personajes intervenciones directamente tomadas del texto de Rojas. Dice Areúsa: "So short our time to pleasure! So quick to turn to sorrow!", lo que evidentemente tiene que ver con las palabras que Melibea dice antes de suicidarse. En la escena final, también, Alfeo y Areúsa, al tiempo, repiten las palabras de Melibea: "His death calls out to mine, calls out to me. I want it now without delay. Calisto! Wait for me, I am coming!" Ante lo cual todos exclaman "Ay" y se cierra la obra. De alguna manera, recreando a través de otros personajes la tragedia de Calisto y Melibea se vuelve a vivir, ya que en ambos casos la obra termina con el lamento por la muerte de Melibea.

En cuanto a su vertiente musical, la ópera la constituyen tres sopranos, dos contraltos, tres bajos, dos tenores y un contratenor acompañados por una viola de gamba. Cabe destacar la presencia de los tres niños que siempre intervienen a la vez, aunque con diferentes voces (los dos contraltos y una soprano), ya que, de alguna manera, es el intento de crear el coro que también había perseguido Nin-Culmell. Asimismo es reseñable la voluntad de recrear el ambiente de la plaza urbana renacentista a través del trabajo de investigación que ha llevado a incorporar, tanto textual como musicalmente, obras de origen sefardí y canciones populares del silgo XVI. Dos de estas obras son efectivamente de origen sefardí. La primera, Majo, con arreglos de V. Couper, es la melodía con la que se abre la obra en voz de las sopranos Templanza y Areúsa y del alto, Otoño, y cuyo eco queda en las palabras de la primera, que repetirá en varias ocasiones "water in the mortar". La segunda, Rahelica baila la canta la soprano que hace de Templanza justo después de que todos los personajes recuerden 
el motivo de las cien monedas y con él los objetivos económicos que mueven a Celestina, Pármeno y Sempronio ${ }^{20}$.

Por otro lado, se incorpora el comienzo de dos obras de la tradición española poético-musical. La primera de ellas, La tricotea, de Alonso ${ }^{21}$, recogida en el Cancionero de Palacio tiene lugar despúes de que Templaza pregunte "And what were Sempronio and Parmeno doing while Celestina was risking her neck?". La segunda, la cantiga de amigo de Martin Códax Cuantas sabedes amar amigo se inserta por primera vez en el momento en el que es mencionado en nombre de Calisto, aunque sólo se trata del primer verso. Posteriormente se repite, después de la confirmación del enamoramiento de Melibea, esta vez en la totalidad que reproducimos ${ }^{22}$.

En esta ópera-instalación podemos ver cómo se mezclan por un lado, elementos de la tradición musical y textual, respetando las palabras de Rojas y reproduciendo melodías populares en su más pura forma, es decir, sin modificar su origen. Por otro lado, la concepción plenamente contemporánea de esta obra, tanto en el formato, como en la estructura lleva a la mezcla de personajes y de lenguajes musicales y textuales, con el uso de sirenas, zumbidos, sollozos, gritos, palmadas, sonidos que se extienden por todo el edificio y golpes con maderas como parte del discurso musical y la integración de personajes heterogéneos como parte de la continuación celestinesca. Tenemos pues, en esta ópera el último ejemplo de una celestinesca entendida en muy amplio sentido como continuación de la obra original ${ }^{23}$.

20 La letra dice así:

Rahelica baila.

Moxonico canta.

Los ratones gordos,

ésos dan las palmas.

Son varias las referencias a lo largo del texto que retoman la idea de Rojas como judío converso y establecen en este hecho el fondo interpretativo de la Tragicomedia. Celestina es calificada, de hecho, dos veces como judía por los demás personajes, Felipe II y el Emperador.

21 El fragmento que recoge la ópera es el siguiente:

La tricotea,

san Martin la vea.

Abres un poc

al agua y señalea.

La bota senbra tuleta,

la señal d'un chapiré,

ge que te gus per mundo spesa,

la botilla plena,

Dama, qui maina,

cerrali la vena.

Orli, çerli, trum, madama,

çerlicer, çerrarli ben,

votr'amí contrari ben,

Niqui, niquidón,

formar gidón, formar gidón.

Yo soy monarchea

de grande nobrea.

Dama, por amor,

dama, bel sé mea.

Dama, yo la vea.

22 En el texto de Young podemos leer estas palabras: "Quantas sabedes amar amigo, / treides comig'a lo mar de Vigo / e banar-nos-emos nas ondas".

23 Pueden verse algunas críticas sobre la recepción de la ópera en: http://www.wsj.com/articles/reviews-of-the-tem- 
En este breve recorrido hemos comprobado la vitalidad de la Tragicomedia de Calisto y Melibea como fuente de inspiración para proyectos musicales escénicos hasta nuestros días. Hemos podido trazar un hilo conductor con sus inevitables saltos desde el primer adaptador, Fernando de Rojas cuando convierte la Comedia en Tragicomedia (o incluso, como muchos pensarán, cuando continúa el primer auto) hasta llegar a la performance que se está llevando a cabo en estos mismos momentos $L a$ Celestina. An installation Opera de la compañía Opera Erratica haciendo, claro está, una detenida parada en los dos textos que consideramos fundamentales por haberse desarrollado dentro de nuestro lenguaje (literario y musical); el de Pedrell y el de Nin-Culmell. Como señalábamos al principio, nuestro objetivo es sentar las bases para poder escribir la historia de La Celestina en su versión de libreto operístico. Claro está, que aún queda por hacer la parte más interesante, que será contrastar las diferentes versiones y, poder, de esta manera, comprobar la versatilidad del motivo, así como la apropiación particular que cada uno de nuestros libretistas aporta al acervo celestinesco, tarea que esperamos poder emprender próximamente.

\section{Obras citadas}

Anderson, Reed, "Calisto \& Melibea. Music by Jerome Rosen and libretto by Edwin Jonig", Celestinesca, 3.2 (1979), pp. 27-31.

Blanco, Emilio "Algunas notas sobre la recepción de La Celestina en los siglos XVI y XVII", en Gregorio Torres ed., Celestina: recepción y herencia de un mito, Universidad de Extremadura, Cáceres, 2001, pp. 17-49.

Bonastre, Francesc, Bonastre i Beltrán, Francesc “El planteamiento operístico de Pedrell”, en E. Casares \& A. Torrente (coords.), La ópera en España e Hispanoamérica: Una creación propia. Madrid: Universidad Complutense e ICCMU, 2001, pp. 187-197.

, "Els models simfònics", Recerca Musicològica, 14-15 (2004-2005), pp. 57-76.

Botta, Patrizia "La Celestina de Palacio en sus aspectos materiales", Boletín de la Real Academia Española, 73 (1993) 25-50 \&347-66.

, "Ancora sulla genesi e paternitá de La Celestina", Cultura neolatina, 44 (1995), pp. 269-283.

, El texto en movimiento (de La Celestina de Palacio a La Celestina posterior", en R. Beltrán y J. L. Canet editores, Cinco Siglos de Celestina: Aportaciones interpretativas, Universidad, Valencia, 1997, pp. 135-160.

Brault, Gerard J., "English translations of the Celestina in the Sixteenth Century", Hispanic Review, 28.4 (1960), pp-161-85.

Conde, Juan Carlos, "El manuscrito II-1520 de la Biblioteca de Palacio y la Celestina: balance y estado de la cuestión", en R. Beltrán y J. L. Canet, eds, Cinco Siglos de Celestina: Aportaciones interpretativas, Universidad, Valencia, 1997, pp. 161-188.

Cortés, Francesc, "La música escénica de Felip Pedrell: Els Pirineus. La Celestina. El Comte Arnau", Recerca Musicologica, 11-12 (1991-1992), pp. 63-97.

, "Ópera española: las obras de Felipe Pedrell”, Cuadernos de música Hispanoamericana, 1 (1996), pp. 187-216.

, "Consideracions sobre els models operístics entre 1875 i 1936", Recerca Musicologica, 14-15 (2004-2005), pp. 77-85. 
Faulhaber, Charles, "Celestina de Palacio: Madrid, Biblioteca de Palacio, Ms1520", Celestinesca, 14.2 (1990), 3-39.

, “Celestina de Palacio: Rojas' Manuscript”, Celestinesca, 15.1 (1991), pp-3-52.

Garcia, Michel, "Consideraciones sobre Celestina de Palacio", Celestinesca, 18.1 (1994), pp. 2-16.

Gereben, Janos, "Note”, San Francisco Classical Voice.

Gimber, Arno, "El estreno mundial y póstumo de la ópera La Celestina de Joaquín Nin-Culmell”, Celestinesca, 33 (2009), pp. 255-258.

Gregori, Josep Maria "Felip Pedrell y el renaixement musical hispànic", Recerca Musicològica, 11-12 (1991-1992), pp. 47-61.

Lobera Serrano, Francisco, "El manuscrito 1520 de Palacio y la tradición impresa de La Celestina", Boletín de la Real Academia Española, 73.258 (1993), pp. 51-67.

Martín Moreno, Antonio, "Felip Pedrell y el descubrimiento del teatro barroco español", Recerca Musicologica, 11-12 (1991-1992), pp. 111-131.

McPheeters, Dean, "La Celestina en el Portugal del siglo XVI", en Manuel Criado del Val ed., La Celestina y su contorno social: actas del I congreso internacional sobre La Celestina, Barcelona, Hispam, 1977, pp. 367-376.

Menéndez Pelayo, Marcelino, Orígenes de la novela IV. Primeras imitaciones de "La Celestina”, Edición nacional de las obras de Menéndez Palayo, Santander, CSIC, 1943. Tanto la nómina de obras como las características de las mismas han sido reseñadas en numerosas ocasiones, razón por la cual no consideramos necesario un tratamiento más profundo en este artículo.

Menéndez Pidal, Ramón, Romancero Hispánico, Madrid, Gredos, 1952.

Montoya Martínez, Jesús, "La presencia de La Celestina en las librerías de finales del siglo XI (uso y consumo de La Celestina)", Celestinesca, 3.1 (1979), pp. 19-32.

Pedrell, Felipe, La Celestina. Tragicomedia lírica de Calisto y Melibea, Barcelona: Tipografía de Salvat, 1903.

, Orientaciones, Paris: P. Ollendorff, 1909.

Snow, Joseph, “La Celestina de Felipe Pedrell”, Celestinesca, 3.1 (1979), pp. 19-32. , "Hacia una historia de la recepción de La Celestina: 1499-1822", Celestinesca, 21 (1997), pp. 115-72.

, "La Tragicomedia de Calisto y Melibea de Juan de Sedeño. Algunas observaciones a su primera escena comparada con la original", Celestinesca, 21 (1997), pp. 13-25.

, "Celestina (1499-1999) Medieval and Modern: Survival \& Renewal of a Spanish Classic", Medieval Prespectives, 1 (2000), pp. 1-11.

, \& Gimber, Arno, "Richard Strauss, Stefan Zweig, Joseph Gregor and the Story of Celestina Opera that Amost was, with a Bibliographical Appendix of Celestina Operas in the Twentieth Century", Celestinesca, 31 (2007), pp. 133-164. 